\title{
Parametric Analysis of Thermal Blooming for High Energy Lasers Considering the Atmospheric Environment
}

\author{
Jawaher Alameri \\ Directed Energy Research Centre \\ Technology Innovation Institute \\ Abu Dhabi, United Arab Emirates \\ jawaher.alameri@tii.ae \\ Chaouki Kasmi \\ Directed Energy Research Centre \\ Technology Innovation Institute \\ Abu Dhabi, United Arab Emirates \\ chaouki.kasmi@tii.ae
}

\author{
Juan Coronel \\ Directed Energy Research Centre \\ Technology Innovation Institute \\ Abu Dhabi, United Arab Emirates \\ juan.coronel@tii.ae \\ Fahad Alyafei \\ Directed Energy Research Centre \\ Technology Innovation Institute \\ Abu Dhabi, United Arab Emirates \\ fahad.alyafei@tii.ae
}

\author{
John Pantoja \\ Directed Energy Research Centre \\ Technology Innovation Institute \\ Abu Dhabi, United Arab Emirates \\ john.pantoja@tii.ae
}

\begin{abstract}
Many studies were dedicated to the analysis of the propagation of High Energy Laser beams in free space. Laser beams propagation performance in the presence of atmospheric constituents may degrade by some interrelated linear and nonlinear phenomena. In this study, it is proposed a self-contained model including non-linear air temperature effect producing thermal distortion in laser propagation. In order to better understand the underlying phenomena, a set of quantifiers are described leading to the characterization of the laser thermal distortion. A parametric study is finally proposed in order to highlight the influence of the different parameters of the simulations.
\end{abstract}

Index Terms-Thermal blooming, thermal distortion, laser propagation, high energy laser.

\section{INTRODUCTION}

The emergence of High Energy Lasers marked a rapid paradigm shift in different research areas [1], [2]. These systems can be implemented in a wide variety of engineering applications from telecommunications, material treatment, welding [3] or defense applications [4]. Recent literature reports achievement of higher than $30 \mathrm{~kW}$ optical power laser demonstrations [5], [6].

High energy laser propagation, especially in the atmosphere, has been a dominant subject in research and development. These studies [7] enabled the evaluation of the laser beam capability to arrive at a desired target (e.g. telescope, vehicle or sample) considering the thermal distortion effects when propagation media temperature increases varying its refractive index. One of the important recorded atmospheric effects is the thermal distortion, known as well as "thermal blooming" [8].

Thermal blooming is the result of nonlinear interaction of the laser radiation waves with the propagation medium constituents. It is produced due to energy dissipation of the

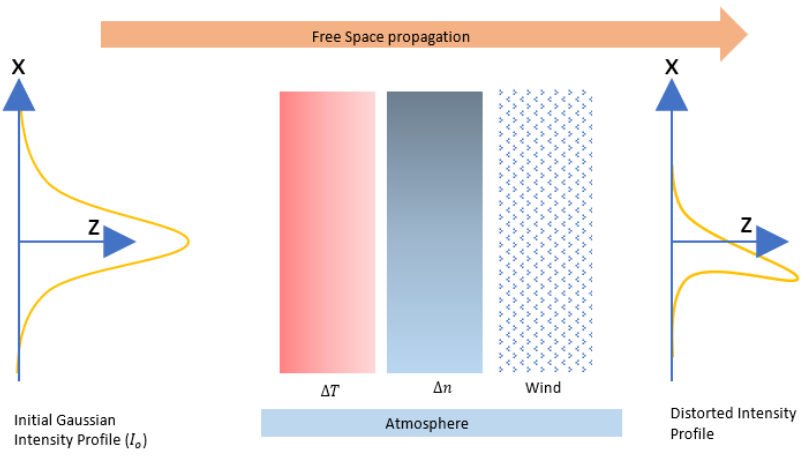

Fig. 1: Thermal blooming schematic concept [8].

laser beam in the medium, which rises its temperature leading to a refractive index variation.

The thermal blooming phenomenon is depicted in Fig. 1. For illustration, the initial propagating beam is assumed with a Gaussian-like profile and a high energy wave. Considering the wave energy, the air surrounding the beam is heated and; consequently, the refractive index of the propagation medium is changed, leading to distortions in the intensity profile. The wind component included on the thermal blooming schematic relates thermal interaction of wind and the temperature variations of the propagation medium refractive index.

Many studies focusing on thermal blooming have been published regarding thermal blooming modeling [9]-[11]. However, these reported results are nor directly reproducible since they do not present all simulation variables considered in their models. Interestingly, it has been observed that nonlinear parameters referring to the propagation were missing. We 
propose hereafter a revision of the proposed formalism adding the non-linearity of the propagation medium by introducing the related parameters. A parametric analysis is also presented, considering the variance of the beam field intensity as figureof-merit for the laser beam characterization when the optical wavelength, wind speed, optical power beam, and beam radius are varied.

The paper is organized as follows. First, the analytical model of the high energy laser beam propagation and the figuresof-merit for laser characterization are detailed in Section 2. Then, in Section 3, the simulations are presented and results are discussed. A parametric analysis is also detailed in this section, in which the influence of the variability of physical parameters on the physical output variables (namely the socalled figures-of-merit) is analyzed. Finally, conclusions are drawn in Section 4.

\section{NumericAl Simulation MODEL}

\section{A. Propagation Algorithm}

The depicted model presented in this section is developed using the paraxial approximation of propagation along $z$ axis under isobaric condition. The heating effects behavior in the atmosphere, considering a complex amplitude electric field and the thermal interaction of electromagnetic wave with air is described using the following differential equations [9]:

$$
\begin{array}{r}
2 i k \frac{\partial E}{\partial z}+\left(\frac{\partial^{2}}{\partial x^{2}}+\frac{\partial^{2}}{\partial y^{2}}+\frac{2 k^{2}}{n} \frac{\partial n}{\partial T} T\right) E=0 \\
\frac{\partial T}{\partial t}+V \frac{\partial T}{\partial x}=\frac{\alpha}{\rho C_{p}} I(x, y, z, t)
\end{array}
$$

Where, $I=(c n) /(8 \pi) E E^{*}$ is the electric field intensity, $k=$ $(2 \pi / \lambda)$ is the wavenumber, $C_{p}$ is the specific heat, $V$ is the medium speed and $n$ represents the medium refractive index.

To obtain a specific solution for Eq. (1) and (2), the initial boundary conditions of the medium should be set to:

$$
E(x, y, 0, t)=E_{0} f(x, y)
$$

where $E_{o}$ represents the initial electric field at the laser output and $f(x, y)$ is the beam intensity profile. Here a Gaussian profile is assumed,

$$
f(x, y)=\exp \left(\frac{-\left(x^{2}+y^{2}\right)}{2}-i\left(\delta x * x^{2}+\delta y * y^{2}\right)\right)
$$

Additionally, the medium boundary conditions are defined as,

$$
\lim _{x, y \rightarrow \pm \infty} E=0 \lim _{\substack{x \rightarrow-\infty \\ y \rightarrow \pm \infty}} T=0
$$

To solve this equation set, normalization [9] is applied to the variables by making $E^{\prime}=E / E_{0}, E_{0}=\sqrt{8 \pi I_{0} /(c n)}, x^{\prime}=$ $x / a, y^{\prime}=y / a, z^{\prime}=z / k a^{2}, t^{\prime}=t / \tau_{v}, \tau_{v}=a / V, T_{0}=$ $n / 2 k^{2} a^{2} \partial n / \partial T, T^{\prime}=T / T 0, I_{0}=P_{0} / \pi a$; where $c$ is the speed of light, $n$ is the propagation medium's refractive index, $a$ is the beam radius, and $P_{0}$ is the initial power of the beam.
From this variable adjustment, Eq. (1) and (2) are organized as follows,

$$
\begin{array}{r}
\frac{\partial E}{\partial z}=\frac{i}{2}\left(\frac{\partial^{2}}{\partial x^{2}}+\frac{\partial^{2}}{\partial y^{2}}+T\right) E \\
\frac{\partial T}{\partial t}+\frac{\partial T}{\partial x}=R I
\end{array}
$$

In Eq. (7), the term $R$ represents the propagation medium nonlinearities. In this work it is proposed to define $R$ considering forced convection conditions and a medium speed much lower than the speed of sound. This leads to $R$ being expressed as follows,

$$
R=\frac{2 \pi}{\lambda} \frac{\alpha a I_{0}}{\rho C_{p} V} n^{\prime} L
$$

where $\alpha$ is the medium absorption coefficient, $C_{p}$ is the specific heat of propagation medium, $\rho$ is the medium's density, $V$ is the propagation medium speed, $n^{\prime}$ is the propagation medium medium refractive index variation with respect to the temperature, and $L$ is the propagation path length.

In addition to this consideration, the parameter $n$ has been estimated based on the isobaric expression for air refractive index from the USA's National Institute of Standards and Technology [12]. This expression includes the atmospheric pressure, relative air humidity, and temperature for a more realistic refractive index value.

To solve Eq. (6) and (7), the values of $E, T, I$ and $f(x, y)$ are set to numerical values and boundary conditions are defined by Eq (4) and (5). According to procedure described by Konyaev et al [9], the system is split using the second order approximation with respect to $z$. In this system, $t$ is set as a fixed variable. The Eq. (6) and (7) are replaced by their equivalent components in $z$ direction. These equations are as follows,

$$
\begin{gathered}
\frac{\partial E_{x}}{\partial z}=\frac{i}{2} \frac{\partial^{2}}{\partial x^{2}} E_{x}, E_{x}(x, y, 0, t)=E(x, y, 0, t) \\
\frac{\partial E_{y}}{\partial z}=\frac{i}{2} \frac{\partial^{2}}{\partial y^{2}} E_{y}, E_{y}(x, y, 0, t)=E_{x}(x, y, \Delta z / 2, t) \\
\frac{\partial E_{z}}{\partial z}=\frac{i}{2} T E_{z}, E_{z}(x, y, 0, t)=E_{y}(x, y, \Delta z / 2, t)
\end{gathered}
$$

$$
\begin{gathered}
\frac{\partial T}{\partial t}+\frac{\partial T}{\partial x}=R I, I(x, y, \Delta z / 2, t)=E_{z} E_{z}^{*}(x, y, \Delta z / 2, t) \\
\frac{\partial E_{y}}{\partial z}=\frac{i}{2} \frac{\partial^{2}}{\partial y^{2}} E_{y}, E_{y}(x, y, \Delta z / 2, t)=E_{x}(x, y, \Delta z, t) \\
\frac{\partial E_{x}}{\partial z}=\frac{i}{2} \frac{\partial^{2}}{\partial x^{2}} E_{x}, E_{x}(x, y, \Delta z / 2, t)=E(x, y, \Delta z, t)
\end{gathered}
$$


Eq. (9),(10),(13) and (14), are solved using Fourier procedure. $E_{x}(x, y)$ and $E_{y}(x, y)$ are solved for $x$ and $y$ coordinates:

$$
\begin{aligned}
& E_{x}(x, y)=\frac{1}{2 \pi} \int_{-\infty}^{\infty} F_{x}\left(x_{x}, y\right) \exp \left(i_{x x} x\right) d x_{x} \\
& E_{y}(x, y)=\frac{1}{2 \pi} \int_{-\infty}^{\infty} F_{y}\left(x, x_{y}\right) \exp \left(i_{x y} y\right) d x_{y}
\end{aligned}
$$

the $F_{x}$ and $F_{y}$ terms represent the spectral Fourier transforms:

$$
\begin{aligned}
& F_{x}(x, y)=\int_{-\infty}^{\infty} E_{x}(x, y) \exp \left(-i_{x x} x\right) d x_{x} \\
& F_{y}(x, y)=\int_{-\infty}^{\infty} E_{y}(x, y) \exp \left(-i_{x y} y\right) d x_{y}
\end{aligned}
$$

This procedure divides the problem into two one-dimensional solution equations:

$$
\begin{aligned}
F_{x}\left(x_{x}, y, 0\right) & =F_{x}\left(x_{x}, y, 0\right) \exp \left(-i x_{x}^{2} \Delta z / 2\right) \\
F_{y}\left(x, x_{x}, \Delta z\right) & =F_{y}\left(x, x_{y}, 0\right) \exp \left(-i x_{x}^{2} \Delta z / 2\right)
\end{aligned}
$$

where $F_{x}$ and $F_{y}$ are the Fourier transform of the $x$ and $y$ components of the electric field.

Following the resolution of Eq. (6) and (7), the field intensity $I$ is deduced by,

$$
I=E E^{*}
$$

Apart from the field intensity computation, it is required to describe the temperature of air while the beam is being propagated. Temperature variations will define thermal lensing distortions in free space and consequently, modifying the laser beam propagation path. The Air temperature along the propagation path is defined as,

$$
\begin{array}{r}
T(x, y, t+\Delta t)=[T(x, y, t)+(\Delta T / \Delta x) T(x-\Delta x, y, t+\Delta t) \\
+\Delta t R I(x, y, t)] /(1+\Delta t / \Delta x) \quad(22)
\end{array}
$$

The framework reference discretization employed in this article to sample $x$ and $y$ is 64 samples each and 128 samples for $z$. Eq. (22) follows the boundary condition defined in Eq. (5).

\section{B. Figures of Merit for Laser Beam}

To observe the thermal distortion effects of a propagating beam in a free space, the intensity field profile distribution is analyzed with respect to two parameters: the energy center of gravity of the intensity profile, and the variance of the intensity field at any point of the propagation path.

1) Energy Center of Gravity: The energy center of gravity of the surface by the intensity field of the propagated beam is expressed as,

$$
\begin{aligned}
x_{c} & =\frac{1}{\pi} \iint x I(x, y) d x d y \\
y_{c} & =\frac{1}{\pi} \iint y I(x, y) d x d y
\end{aligned}
$$

Where $x$ and $y$ are the Cartesian coordinates of the intensity field surface. The energy center of gravity of this surface is expressed as the coordinates $x_{c}$ and $y_{c}$. The energy center of gravity moves away from the initial point (for a Gaussian profile it is 0,0$)$ as it is distorted along the propagation path.
2) Intensity Field Variance: The variance of the intensity field distribution for $x$ and $y$, are represented by $\sigma_{x}^{2}$ and $\sigma_{x}^{2}$, respectively. These are expressed as [10],

$$
\begin{aligned}
\sigma_{x}^{2} & =\int_{-\infty}^{\infty}\left(x-x_{c}\right)^{2} I(x, y) d x d y \\
\sigma_{y}^{2} & =\int_{-\infty}^{\infty}\left(y-y_{c}\right)^{2} I(x, y) d x d y
\end{aligned}
$$

This parameter represents the squared variation of the field intensity from its mean. In other words, the variance is a measure of the intensity field uniformity.

\section{Simulation Results}

Numerical simulations were implemented using the physical parameters presented in Table I. These set of parameters are kept constant along the parametric analysis performed in this work. On the other hand, the variables considered in the parametric analysis are: laser beam power $P_{o}$, laser wavelength $\lambda$, wind speed $V$, and beam radius $a$. The parametric input variables are summarized in Table II, as well as the outcome variables to be analyzed. In this work we have considered three parametric analysis possibilities: (i) the variation of the optical beam power and the uncertainty related to the wavelength, (ii) the optical power beam constant with wind speed sweep, and (iii) the high energy laser beam radius sweep.

TABLE I: Physical parameters for numerical simulations.

\begin{tabular}{|l|l|l|}
\hline Parameter & Value & Units \\
\hline \hline Propagation path length $(L)$ & 15 & $m$ \\
\hline Air refractive index $(n)$ & 1.0003 & - \\
\hline Air specific heat $\left(C_{p}\right)$ & $1.005 \times 10^{-3}$ & $J / K$ \\
\hline Absorption air coeff. $(\alpha)$ & $3 \times 10^{-2}$ & $1 / m$ \\
\hline
\end{tabular}

TABLE II: Input parameter value range.

\begin{tabular}{|l|l|l|}
\hline Variable & Description & Range \\
\hline \hline Optical power beam & $P_{o}$ & $1-4 \mathrm{~kW}$ \\
\hline Laser beam radius & $a$ & $0.009-0.011 \mathrm{~m}$ \\
\hline Optical wavelength & $\lambda_{o}$ & $1040 \mathrm{~nm} \pm 10 \%$ \\
\hline Wind Speed & $V$ & $10-40 \mathrm{~m} / \mathrm{s}$ \\
\hline
\end{tabular}

\section{A. Variation of the optical beam power}

For this analysis, the parametric sweep consists of four $P_{o}$ values $1,2,3$, and $4 \mathrm{~kW}$, and three wavelength values: $\pm 10 \%$ $\lambda_{o}$ and $\lambda_{o}$, these values are presented in Table I. Additionally, the outcomes of this model are the electric field intensity $I$ in the propagation plane, the energy center of gravity, the intensity variance in $x$ and $y, \sigma_{x}^{2}$ and $\sigma_{y}^{2}$, and the maximum intensity to the initial intensity ratio $\operatorname{Imax} / I_{0}$. 
1) $1 \mathrm{~kW}$ Laser Beam Simulation Results: The numerical thermal blooming simulation for $1 \mathrm{~kW}$ laser beam was performed according to the previous parametric sweep description. Fig. 2 presents the intensity profile distribution for $1040 \mathrm{~nm}$ wavelength. The intensity profile presents distortions from the Gaussian beam as the focal plane is moved towards longer distance. Regarding the energy center of gravity of the intensity profile at the focal plane (marked as ' $*$ ' on the $2 \mathrm{D}$ plot), a displacement of this point as the intensity surface gets deformed by the thermal distortion is evidenced. In Fig. 3 it is observed a more detailed approach for the energy center of gravity of each intensity surface for different wavelength values. From Fig. 3 we can obtain more insights about the energy center of gravity of the intensity surface, as long as the focal distance increases, the coordinate values $\left(x_{c}, y_{c}\right)$ moves from the origin towards higher values in $x$ and $y$ axis. It is important to notice that $x$ coordinate of the energy center of gravity remains almost the same value for different ranges. This small difference value could be attributed to the numerical method itself considering the discretization performed in this study. However, for $y$ coordinate the variation for the simulation range reaches $0.0192 \mathrm{~cm}$. Additionally this behavior is accentuated when the beam wavelength is smaller.
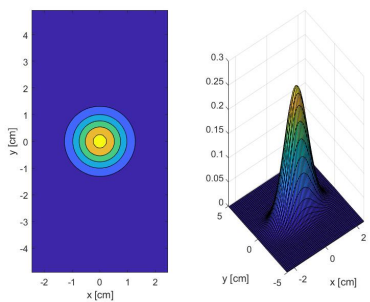

(a) $0 \mathrm{~m}$.
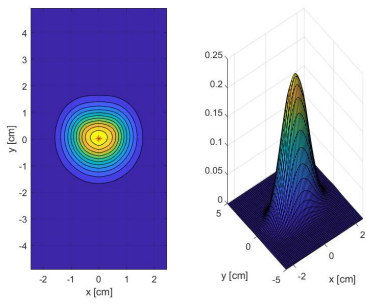

(c) $10 \mathrm{~m}$.
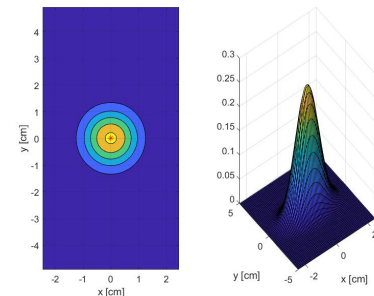

(b) $5 \mathrm{~m}$.
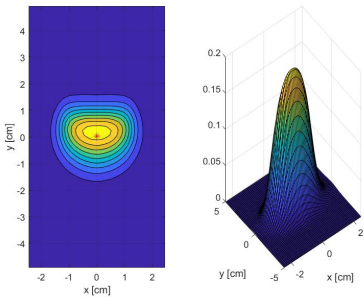

(d) $15 \mathrm{~m}$.
Fig. 2: $1 \mathrm{~kW}$ intensity profile for different propagation length values.

2) $2 \mathrm{~kW}$ Laser Beam Simulation Results: The numerical simulation results when the high energy laser beam power is set to $2 \mathrm{kw}$ are shown in Fig. 4. From this figure it is evident that thermal distortion effects as the laser beam power increases are stronger when compared to the $1 \mathrm{~kW}$ simulation. The coordinates of the energy center of gravity for this case are shown in Fig. 5. For $x_{c}$ coordinate, contrary to what is seen in $1 \mathrm{~kW}$ scenario, the variation of the $x_{c}$ is evident along the path length increase. $x_{c}$ variation escalates when the laser wavelength increases by $10 \%(1144 \mathrm{~nm})$. From the performed

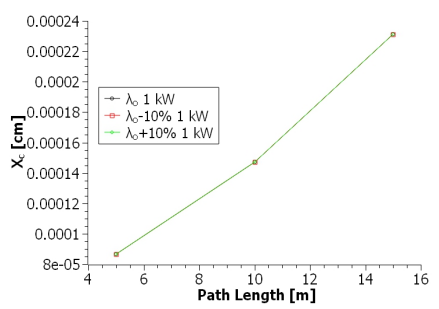

(a) $x_{c}$.

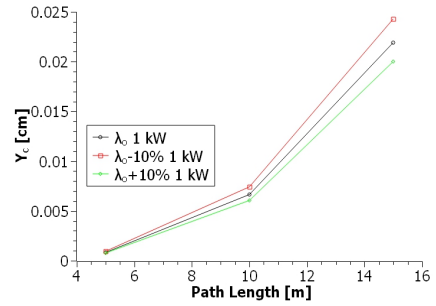

(b) $y_{c}$.
Fig. 3: Energy center of gravity coordinates for $1 \mathrm{~kW}$ laser beam simulation.

simulation at $15 \mathrm{~m}$ path length, a difference of $2.7 \times 10^{-4} \mathrm{~cm}$ is observed. Furthermore, regarding to $y_{c}$ coordinate value, it is inferred from Fig. 5b that the energy center of gravity moves away from the origin for lower wavelength $(936 \mathrm{~nm})$.
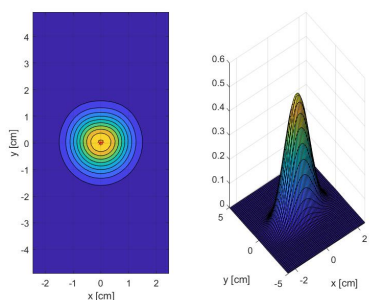

(a) $5 \mathrm{~m}$

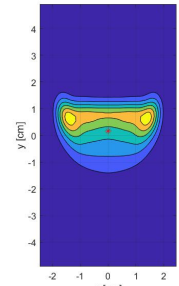

(c) $15 \mathrm{~m}$.
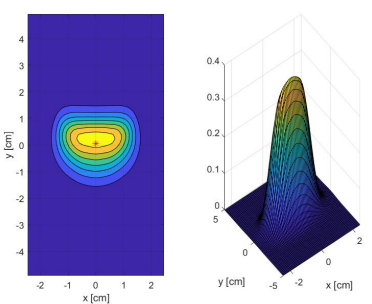

(b) $10 \mathrm{~m}$.

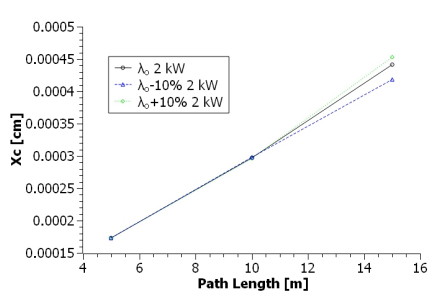

(a) $x_{c}$.

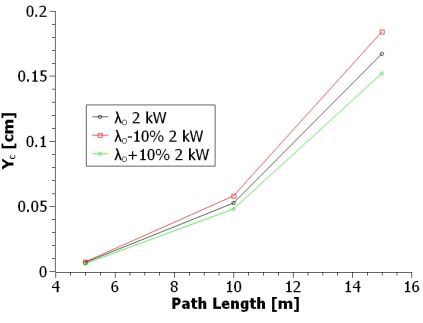

(b) $y_{c}$.
Fig. 5: Energy center of gravity coordinates for $2 \mathrm{~kW}$ laser beam simulation.

3) $3 \mathrm{~kW}$ Laser Beam Simulation Results: The simulation results for $3 \mathrm{~kW}$ laser beam are shown in Fig. 6. The thermal distortion effects increase as the optical power beam does. In Fig. $6 \mathrm{c}$, the intensity profile at $15 \mathrm{~m}$ is highly distorted with 
respect to the ideal Gaussian-like intensity field distribution. The energy center of gravity of the intensity profile surface for 966, 1040 and $1144 \mathrm{~nm}$ are shown in Fig. 7. From this figure it is observed that the coordinate $x_{c}$ value increases as the path length becomes larger. Additionally, the $x_{c}$ coordinate variation increases as the beam wavelength decreases by $10 \%$, see Fig. 7a. The total variation of $x_{c}$ coordinate is $0.041 \mathrm{~cm}$.

In $y_{c}$ coordinate, a similar trend to that one observed for $x_{c}$ coordinate is shown. $y_{c}$ varies in proportion to the path distance, see Fig. 6c. It is inferred that $y_{c}$ has a difference of $0.071 \mathrm{~cm}$ when simulated for $966 \mathrm{~nm}$ and $1144 \mathrm{~nm}$.
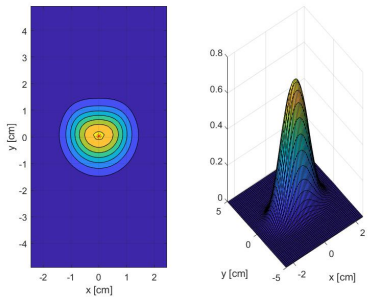

(a) $5 \mathrm{~m}$.

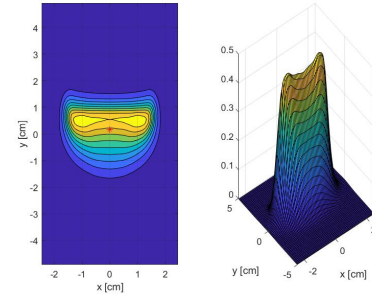

(b) $10 \mathrm{~m}$.
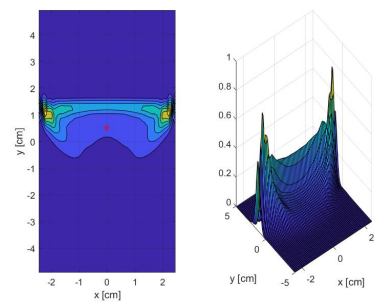

(c) $15 \mathrm{~m}$.

Fig. 6: $3 \mathrm{~kW}$ intensity profile for different propagation length values.

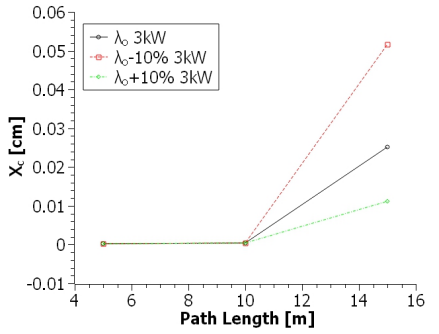

(a) $x_{c}$.

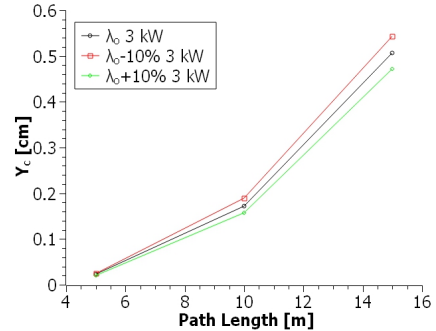

(b) $y_{c}$.
Fig. 7: Energy center of gravity coordinates for $3 \mathrm{~kW}$ laser beam simulation.

4) $4 \mathrm{~kW}$ Laser Beam Simulation Results: The $4 \mathrm{~kW}$ laser beam power simulation results at a wavelength of $1040 \mathrm{~nm}$ are shown in Fig. 8. Comparing the intensity profile distribution for the $1 \mathrm{~kW}$ laser beam at $5 \mathrm{~m}$ shown in Fig. $2 \mathrm{~b}$ with the $4 k W$ case presented in Fig. 8a, it is clearly evident that the beam optical power is inversely proportional to the profile distribution degradation when the beam is analyzed at the same distance $(5 \mathrm{~m})$. Furthermore, the energy center of gravity of the intensity field for coordinate $x_{c}$ is presented in Fig. 9. Moreover, the $y_{c}$ coordinate values are directly proportional to the path length. But, inversely proportional to the beam wavelength, as seen in Fig. $9 b$.
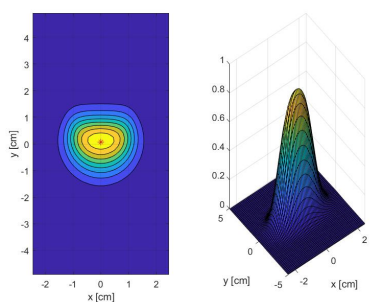

(a) $5 \mathrm{~m}$.
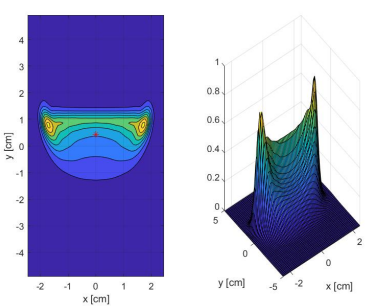

(b) $10 \mathrm{~m}$
Fig. 8: $4 k W$ intensity profile for different focal length.

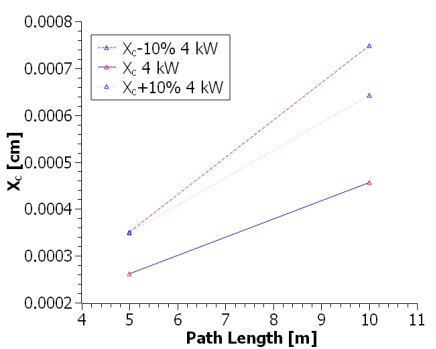

(a) $x_{c}$.

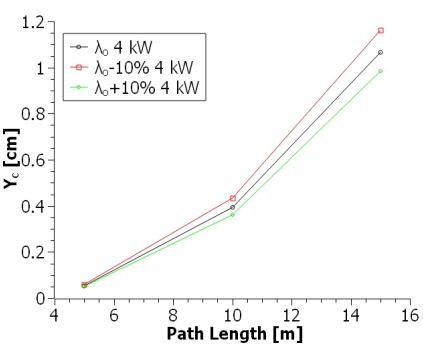

(b) $y_{c}$.
Fig. 9: Energy center of gravity coordinates for $4 \mathrm{~kW}$ laser beam simulation.

\section{B. Intensity Beam Quality Comparison}

The quality of the laser beam under thermal blooming conditions is analyzed with the intensity field variance defined in Eq. (25) and (26). Fig. 10 presents $\sigma_{x}^{2}$ and $\sigma_{y}^{2}$ values for each optical beam power value and their corresponding wavelengths discussed in the previous section. The increase of variance shows higher intensity beam perturbations due to thermal blooming.

The trend in the variance behavior is kept when optical power and distance increase. The highest obtained variance is observed for the $4 \mathrm{~kW}$ simulation scenario. This is consistent if it is noticed that the highest intensity profile distortion occurs at this optical power value, as seen in Fig. 8.

From simulation results, the degradation of the intensity profile variance with the beam wavelength is expected considering the influence of the beam wavelength in the non-linearity propagation medium $R$ described in Eq. (8).

The uniformity of the laser beam can be observed by plotting the $I \max / I_{o}$ curve for each optical power and wavelength parameter. Fig. 11 presents the $\operatorname{Imax} / I_{o}$ ratio for all simulation possibilities under this scenario. It is clearly observed from this figure that the considered cases reach their peaks at the optical path length highest value, especially when the beam wavelength is reduced by $10 \%$. This is explained by the increase of the $R$ parameter, which leads 


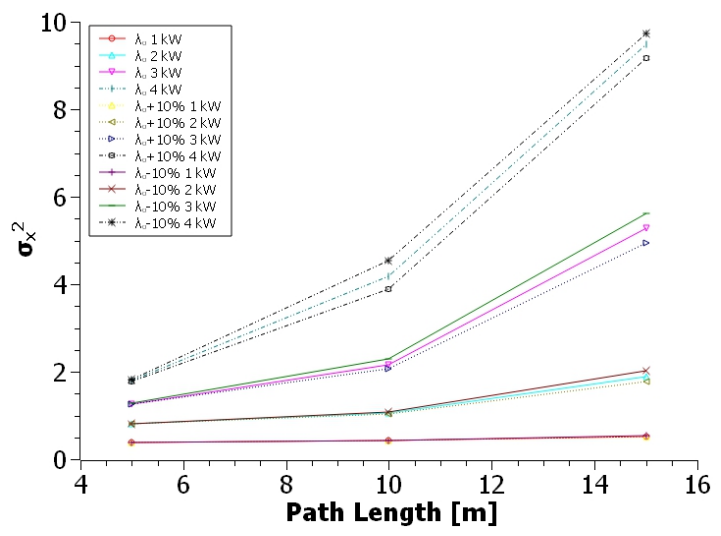

(a) $\sigma_{x}^{2}$.

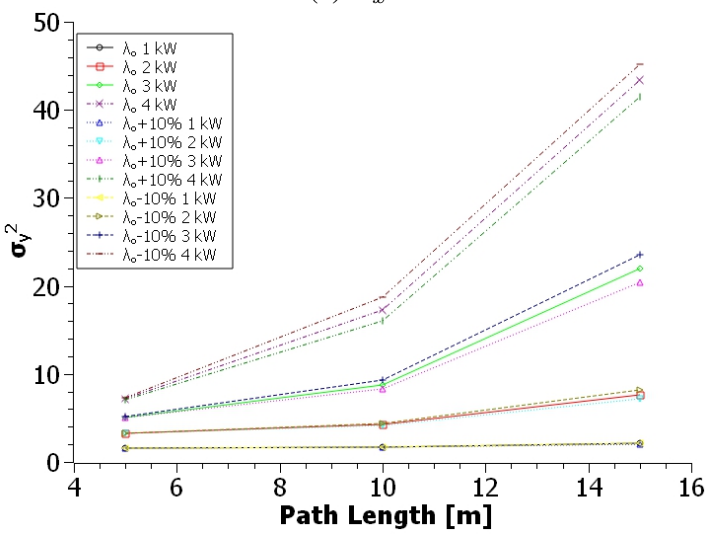

(b) $\sigma_{y}^{2}$.

Fig. 10: Optical beam intensity field variance in $x$ and $y$ axis.

to a higher thermal distortion of the intensity beam. As such, $R$ is inversely proportional to $\lambda$.

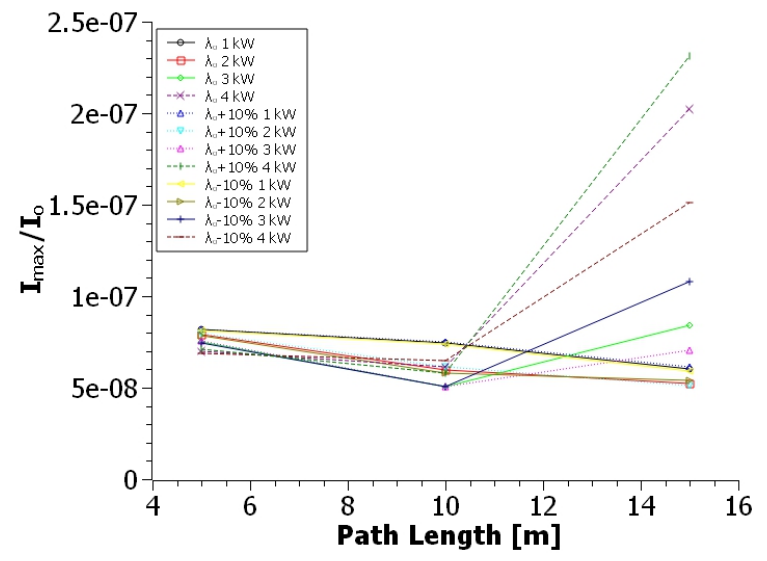

Fig. 11: $\operatorname{Imax} / I_{o}$ ratio for different optical power values and wavelength for the performed simulations.

\section{Variation of wind speed}

Under this analysis conditions, the numerical simulations are performed with wind speed values from 10 to $40 \mathrm{~m} / \mathrm{s}$ (5 $\mathrm{m} / \mathrm{s}$ steps) with an optical power of $2 \mathrm{~kW}$. Fig. 12 presents the intensity profile for the 12 possible simulation conditions. It is clearly observed in this figure that as wind speed increases, the thermal distortion effects decrease. This is a consistent result since the air moving faster will not get heated enough for significant thermal distortion. This is supported as well by the non-linearity medium constant shown in Eq. (8), in which wind speed $(V)$ is inversely proportional to $R$.

In Fig. 12, the energy center of gravity of the intensity surface is marked with the symbol ' $*$ '. It moves closer to the origin as long as the wind speed increases. This is followed by a reduction of the distortion of the intensity profile. The coordinates of the energy center of gravity are presented in Fig. 13. From this figure it is inferred that the energy center of gravity coordinate $y_{c}$ is higher than $x_{c}$ values. This is explained by the direction of wind arriving in $x$ direction that produces symmetric perturbations in $y$ axis. The partial slopes of the curves obtained for the energy center of gravity as a function of the path length are shown in Table III. In addition

TABLE III: Slope of $x_{c}$ and $y_{c}$ for wind speed parametric simulation.

\begin{tabular}{|l|l|l|l|}
\hline Wind speed & Range & $x_{c}$ slope & $y_{c}$ slope \\
\hline \hline $10 \mathrm{~m} / \mathrm{s}$ & $5-10 \mathrm{~m}$ & $2.59 \times 10^{-7}$ & $1.78 \times 10^{-4}$ \\
\hline $10 \mathrm{~m} / \mathrm{s}$ & $10-15 \mathrm{~m}$ & $1.14 \times 10^{-5}$ & $4.13 \times 10^{-4}$ \\
\hline $20 \mathrm{~m} / \mathrm{s}$ & $5-10 \mathrm{~m}$ & $2.47 \times 10^{-7}$ & $9.14 \times 10^{-5}$ \\
\hline $20 \mathrm{~m} / \mathrm{s}$ & $10-15 \mathrm{~m}$ & $2.47 \times 10^{-7}$ & $9.14 \times 10^{-5}$ \\
\hline $30 \mathrm{~m} / \mathrm{s}$ & $5-10 \mathrm{~m}$ & $2.87 \times 10^{-7}$ & $2.28 \times 10^{-4}$ \\
\hline $30 \mathrm{~m} / \mathrm{s}$ & $10-15 \mathrm{~m}$ & $2.45 \times 10^{-7}$ & $6.15 \times 10^{-5}$ \\
\hline $40 \mathrm{~m} / \mathrm{s}$ & $5-10 \mathrm{~m}$ & $2.43 \times 10^{-7}$ & $4.63 \times 10^{-5}$ \\
\hline $40 \mathrm{~m} / \mathrm{s}$ & $10-15 \mathrm{~m}$ & $3.42 \times 10^{-7}$ & $1.19 \times 10^{-4}$ \\
\hline
\end{tabular}

to the intensity field distribution approach, the variance for $x$ and $y$ axes is computed, represented by $\sigma_{x}^{2}$ and $\sigma_{y}^{2}$ respectively. These are shown in Fig. 14. The variance of the intensity beam in $x$ and $y$ decreases when wind speed decreases. In $x$ and $y$ axes, the intensity variance decreases by $40 \%$ for $15 \mathrm{~m}$ when the wind speed increases from 10 to $40 \mathrm{~m} / \mathrm{s}$. However, the variance magnitude $\sigma_{y}^{2}$ axis is higher than $\sigma_{x}^{2}$.

From these results it is inferred that the thermal distortion can be softened by adjusting laser parameters in order to reduce the $R$ magnitude. From Eq. (8) wavelength $(\lambda)$, propagation path length $(L)$ and beam radius can be set to more convenient values as a natural compensation mechanism. However, there are some applications where this is not possible as in outdoor applications where intrinsic air properties (molecule resonance at certain wavelength ranges) deteriorates the beam propagation capability. It is clearly outlined in the first parametric scenario presented in this article that wavelength reduction affects the non-linear response of the medium. 


\section{Variation of optical beam radius}

In this parametric analysis, the optical beam radius is swept from 0.9 to $1.1 \mathrm{~cm}(0.1 \mathrm{~cm}$ step) to observe the influence of the thermal blooming. The simulation results for the intensity beam distribution are shown in Fig. 15. In this figure it is noticed that the thermal distortion effects reduce when the optical beam size is increased. This is verified in our model by taking into account the non-linearity parameter $R$ is directly proportional to the laser initial intensity $I_{o}$. The laser intensity increases when the beam surface size is reduced (lower radius). This is why the thermal distortion effects increase at $0.9 \mathrm{~cm}$ radius.

The energy center of gravity coordinates for this parametric simulation are plotted in Fig. 16. The coordinates $x_{c}$ and $y_{c}$ curves slopes are presented in Table IV. From this table it is observed that $x_{c}$ slope increases as the beam radius is reduced. The slope becomes steeper for the 10 to $15 \mathrm{~m}$ range. Similar results are obtained with $y_{c}$.

The variance $\sigma_{x}^{2}$ and $\sigma_{y}^{2}$ of the intensity field is computed in this work as a figure-of-merit. These values are plotted in Fig. 17 for $x$ and $y$ axes. As previously mentioned, Fig. 17a and Fig. $17 \mathrm{~b}$ confirms that $\sigma_{x}^{2}$ and $\sigma_{y}^{2}$ degrade as the beam radius decreases. For $15 \mathrm{~m}$ range, the degradation of $\sigma_{x}^{2}$ is $44.5 \%$

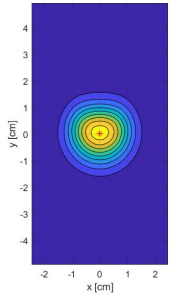

(a) $5 \mathrm{~m}$ at $10 \mathrm{~m} / \mathrm{s}$.
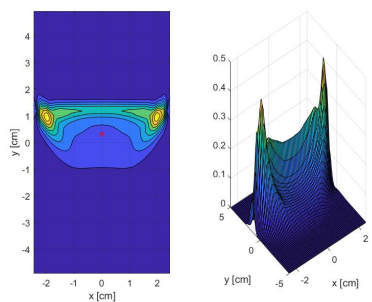

(c) $15 \mathrm{~m}$ at $10 \mathrm{~m} / \mathrm{s}$.
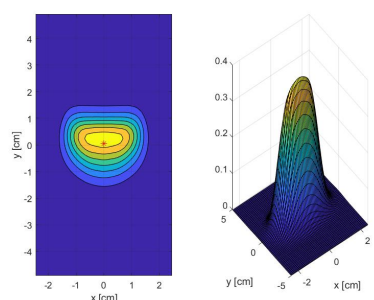

(e) $10 \mathrm{~m}$ at $20 \mathrm{~m} / \mathrm{s}$.
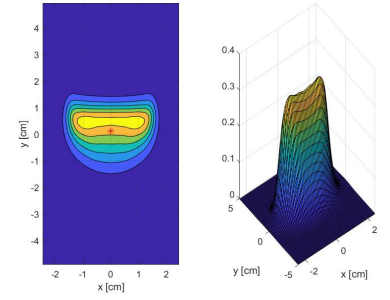

(b) $10 \mathrm{~m}$ at $10 \mathrm{~m} / \mathrm{s}$.
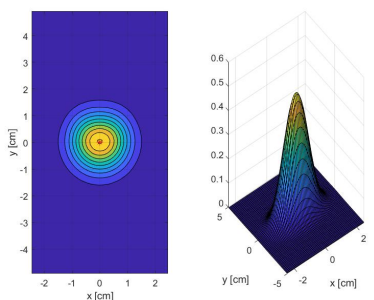

(d) $5 \mathrm{~m}$ at $20 \mathrm{~m} / \mathrm{s}$.
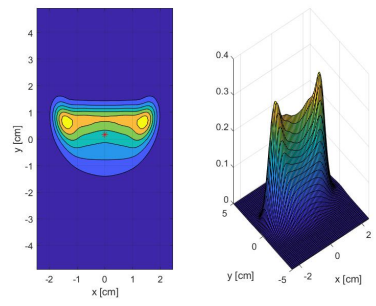

(f) $15 \mathrm{~m}$ at $20 \mathrm{~m} / \mathrm{s}$.
Fig. 12: Intensity profile for $2 \mathrm{~kW}$ laser beam at different wind speed.

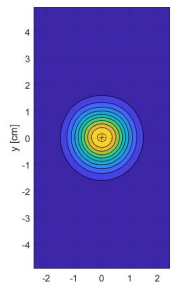

$\times$

(g) $5 \mathrm{~m}$ at $30 \mathrm{~m} / \mathrm{s}$.
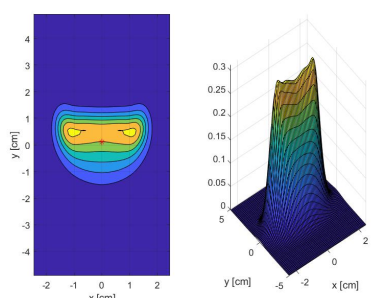

(i) $15 \mathrm{~m}$ at $30 \mathrm{~m} / \mathrm{s}$.
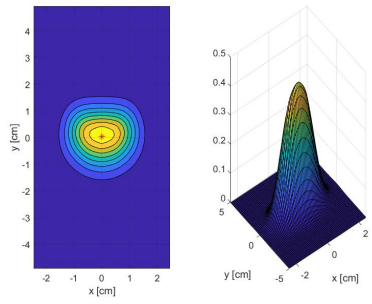

(k) $10 \mathrm{~m}$ at $40 \mathrm{~m} / \mathrm{s}$.
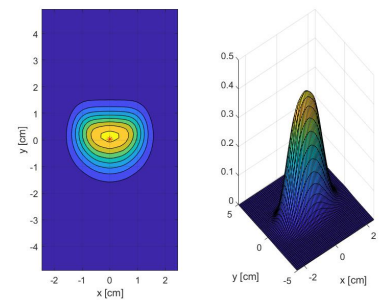

(h) $10 \mathrm{~m}$ at $30 \mathrm{~m} / \mathrm{s}$.
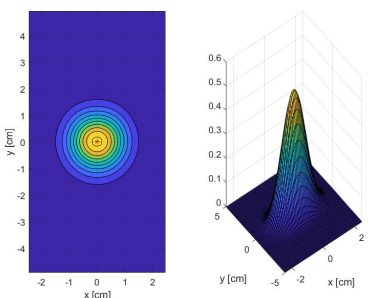

(j) $5 \mathrm{~m}$ at $40 \mathrm{~m} / \mathrm{s}$.
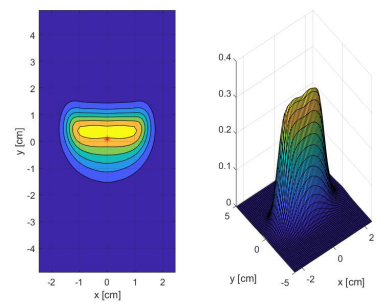

(1) $15 \mathrm{~m}$ at $40 \mathrm{~m} / \mathrm{s}$.
Fig. 12: Intensity profile for $2 \mathrm{~kW}$ laser beam at different wind speed (cont).

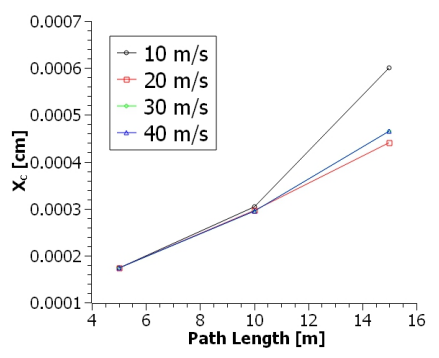

(a) $x_{c}$.

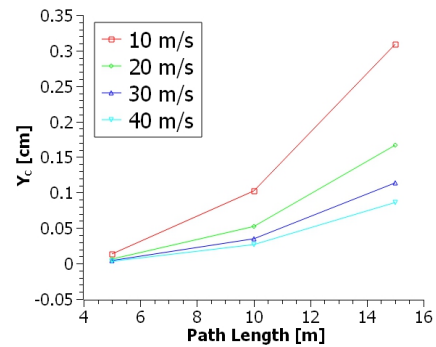

(b) $y_{c}$.
Fig. 13: Energy center of gravity coordinates for wind variation parametric analysis.

when the radius is reduced in $2 \mathrm{~mm}$, and for $\sigma_{y}^{2}$ this is $44 \%$ for the same radius variation.

As observed in this parametric simulation, beam radius variation is a good strategy to mitigate thermal blooming effects. All free space propagation is highly influenced by the non-linearity parameter of the medium $R$. However, the radiation dose $\left(\mathrm{kW} / \mathrm{cm}^{2}\right)$ reduction to attenuate thermal blooming effects might affect the laser performance in directed energy applications. Depending on the application the high energy laser beam, a trade-off between these parameters must be found to assure the performance requirements of the laser. 


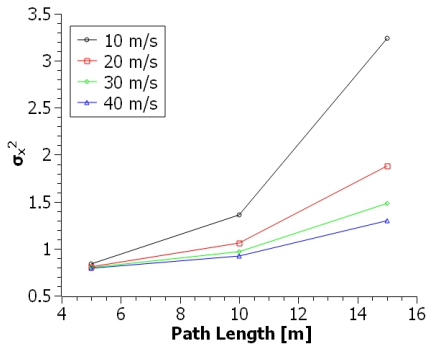

(a) $\sigma_{x}^{2}$.

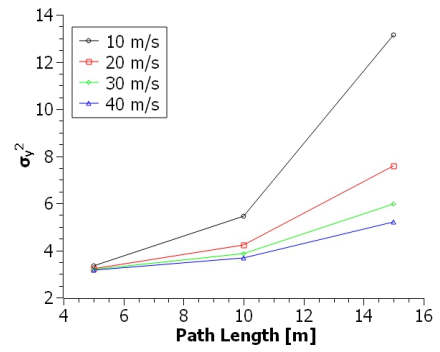

(b) $\sigma_{y}^{2}$.
Fig. 14: Variance $\sigma_{x}^{2}$ and $\sigma_{y}^{2}$ for different wind speed values.

TABLE IV: Slope of $x_{c}$ and $y_{c}$ for radius parametric simulation.

\begin{tabular}{|l|l|l|l|}
\hline Radius & Range & $x_{c}$ slope & $y_{c}$ slope \\
\hline \hline $0.9 \mathrm{~cm}$ & $5-10 \mathrm{~m}$ & $3.10 \times 10^{-7}$ & $1.53 \times 10^{-4}$ \\
\hline $0.9 \mathrm{~cm}$ & $10-15 \mathrm{~m}$ & $1.58 \times 10^{-7}$ & $3.74 \times 10^{-4}$ \\
\hline $1 \mathrm{~cm}$ & $5-10 \mathrm{~m}$ & $2.47 \times 10^{-7}$ & $9.14 \times 10^{-5}$ \\
\hline $1 \mathrm{~cm}$ & $10-15 \mathrm{~m}$ & $2.87 \times 10^{-7}$ & $2.28 \times 10^{-4}$ \\
\hline $1.1 \mathrm{~cm}$ & $5-10 \mathrm{~m}$ & $2.03 \times 10^{-7}$ & $5.71 \times 10^{-5}$ \\
\hline $1.1 \mathrm{~cm}$ & $10-15 \mathrm{~m}$ & $2.75 \times 10^{-7}$ & $1.45 \times 10^{-4}$ \\
\hline
\end{tabular}

\section{CONCLUSIONS}

The implementation of an algorithm has been proposed allowing the study of the thermal blooming in high energy lasers free space propagation. Additionally, the non-linearity medium parameter of the propagation medium, air in our case, has been introduced. The non-linearity of the medium term takes into account some intrinsic characteristics of air like the attenuation of the beam (i.e absorption) and the specific heat in order to simulate a correct beam-air interaction.

A parametric simulation was implemented considering the following variables: the beam power expansion, the wind speed variation, and the beam surface size variation. It has been deduced that the increase of the laser optical beam power and the thermal blooming effects are accentuated. The amount of energy induced to the medium is directly proportional to the surrounding air temperature. The wind speed increase helps in thermal blooming effects reduction. This influence reduction is explained by the non-linearity of the medium and, the inversely proportional nature of wind speed with respect to $R$. Additionally, the reduction of beam radius increases the thermal blooming effects as consequence of the increase of the laser intensity radiation in a small surface.

Interestingly, it has been observed that the manipulation of parameters could lead to the reduction of the thermal blooming. Nevertheless, it is obvious that this technique would reach high limitations due to the complexity to manipulate the laser beam at long distance. In contrary, specific strategies such as compensation techniques through adaptive optics have been proposed in the literature. Future work, will be dedicated to
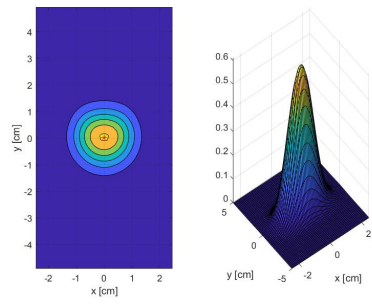

(a) radius $0.9 \mathrm{~cm}$ at $5 \mathrm{~m}$.
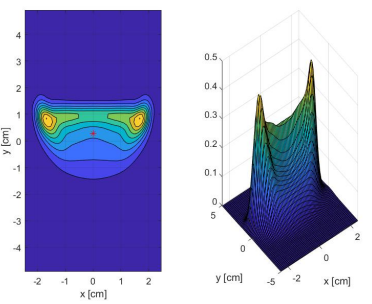

(c) radius $0.9 \mathrm{~cm}$ at $15 \mathrm{~m}$.
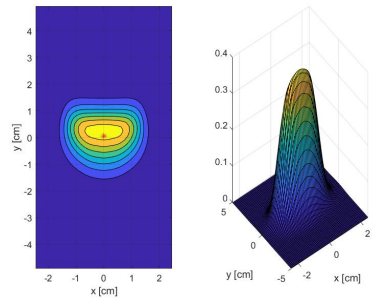

(e) radius $1 \mathrm{~cm}$ at $10 \mathrm{~m}$.
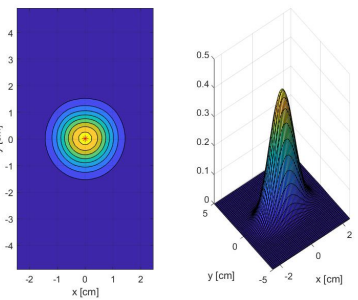

(g) radius $1.1 \mathrm{~cm}$ at $5 \mathrm{~m}$.

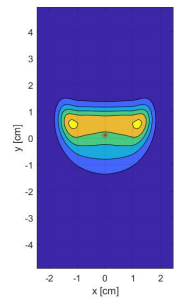

(i) radius $1.1 \mathrm{~cm}$ at $15 \mathrm{~m}$.

Fig. 15: Intensity profile for $2 k W$ laser beam for different beam size.

compensation techniques and their introducing in the presented model. Experimental validation of the proposed approach is also in progress. 


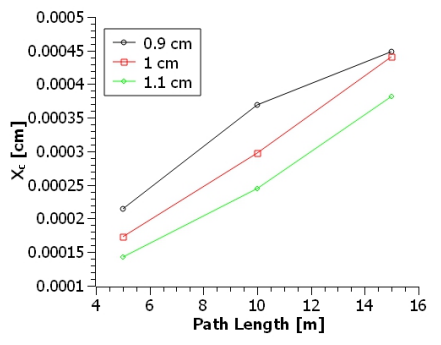

(a) $x_{c}$.

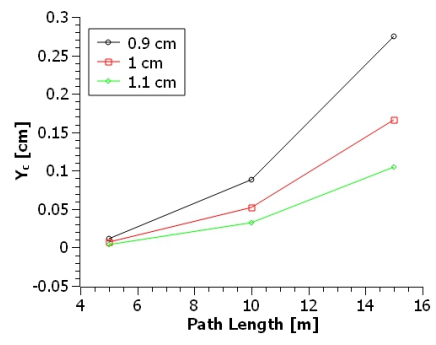

(b) $y_{c}$.

Fig. 16: Coordinates of the energy center of gravity for the beam radius variation parametric simulation.

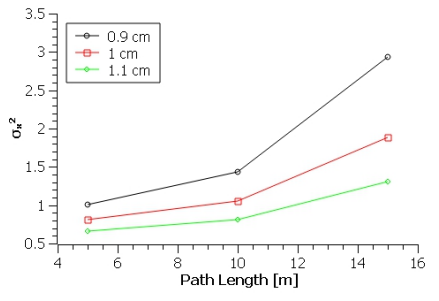

(a) $\sigma_{x}^{2}$.

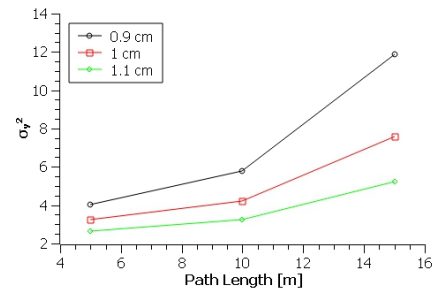

(b) $\sigma_{y}^{2}$.

Fig. 17: Variance $\sigma_{x}^{2}$ and $\sigma_{y}^{2}$ for different radius values.

\section{ACKNOWLEDGMENT}

Authors would like to thank Juan Galvis and Dr. Felix Vega from the Directed Energy Research Centre at Technology Innovation Institute for their support in the analytical simulation and feed-backs on this work.

\section{REFERENCES}

[1] M. N. Zervas and C. A. Codemard, "High Power Fiber Lasers: A Review," IEEE Journal of Selected Topics in Quantum Electronics, vol. 20, no. 5, pp. 219-241, 9 2014. [Online]. Available: http://ieeexplore.ieee.org/document/6808413/

[2] J. Hecht, "Short history of laser development," Optical Engineering, vol. 49, no. 9, p. 091002, 92010.

[3] —-, "High-Power Fiber Lasers," Optics and Photonics News, no. October, pp. 30-37, 2018. [Online]. Available: shorturl.at/gkxEV

[4] P. Sprangle, B. Hafizi, A. Ting, R. P. Fischer, C. C. Davis, and W. Nelson, "High-power lasers for directed-energy applications: reply," Applied Optics, vol. 56, no. 16, p. 4825, 2017.

[5] M. Jung, T. Riesbeck, J. Schmitz, T. Baumgärtel, K. Ludewigt, and A. Graf, "High energy laser demonstrators for defense applications," XXI International Symposium on High Power Laser Systems and Applications 2016, vol. 10254, no. January 2017, p. 1025416, 2017.

[6] J. Cook, "High-energy laser weapons since the early 1960s," Optical Engineering, vol. 52, no. 2, p. 021007, 2012.

[7] B. Zohuri, Directed Energy Weapons. Cham: Springer International Publishing, 2016. [Online]. Available: http://link.springer.com/10.1007/978-3-319-31289-7

[8] F. G. Gebhardt, "Twenty-five years of thermal blooming: an overview," Propagation of High-Energy Laser Beams Through the Earth's Atmosphere, vol. 1221, no. May 1990, p. 2, 1990.

[9] P. Konyaev and V. Lukin, "Thermal distortions of focused laser beams in the atmosphere," Applied Optics, vol. 24, no. 3, p. 415, 2 1985. [Online]. Available: https://www.osapublishing.org/abstract.cfm?URI=ao24-3-415

[10] M. W. Sasnett and T. J. Johnston, "Beam characterization and measurement of propagation attributes," in proc. SPIE, R. N. Hindy and Y. Kohanzadeh, Eds., vol. 1414, no. 1414, 5 1991, pp. 21-32. [Online]. Available: http://proceedings.spiedigitallibrary.org/proceeding. aspx ?articleid=957485

[11] V. A. Banakh and A. V. Falits, "Numerical simulation of propagation of laser beams formed by multielement apertures in a turbulent atmosphere under thermal blooming," Atmospheric and Oceanic Optics, vol. 26, no. 6, pp. 455-465, 11 2013. [Online]. Available: http://link.springer.com/10.1134/S102485601306002X

[12] NIST, "Simple Shop-floor Formula for Refractive Index of Air." [Online]. Available: https://emtoolbox.nist.gov/Wavelength/Documentation.aspAppendixB 